\title{
STUDY OF THE DYNAMICS OF PHYSICAL QUALITIES OF STUDENTS OF BASKETBALL IN THE ANNUAL MACROCYCLE
}

\author{
B. Zauranbekov, L. Kudashov, N. Kaefer, \\ ZT Andreyushkin, I. L., Kudashov, E. S. \\ Kazakh Academy of sports tourism, the country of Kazakhstan, Almaty
}

\begin{abstract}
Summary
The relevance of the chosen topic. The article considers the problem of training basketball players in high school that is actual that requires special scientific developments and approaches to training and team building of the University subject to the time limit of their study at the University and on exercise, while the proposed scientific and methodological literature approaches to the training process is not always suitable for these teams. Analysis of the performance coaches at the University showed that the focus they concentrate on technical and tactical training of students, and the questions of development of physical qualities is usually sidelined. The composition of the student basketball team changes all the time, making it difficult to pick the team on the field.

Given the urgency of the problem was implemented following the aim is to justify the effectiveness of management training for basketball players in training at the University on the basis of testing in the training process of exercises for development of physical qualities of basketball players and to evaluate their dynamics in the annual macrocycle, depending on the characteristics of the periods of training and physiological capabilities of the organism.

In the experimental group in accordance with the annual schedule of the training process is composed of sets of exercises for speed-strength training, which was included in the structure of the annual macrocycle. Was developed by exercises that affect the development of weak muscle groups and to master the technique of special strength exercises.

During the period of experimental training occurred the greatest increase in running 20 meters, at 60 meters and 20 meters with dribbling the ball, when moving into a protective stance, long jump, high jump, Shuttle run $2 \times 40$, test of Cooper, serial jumping, throwing the medicine ball.
\end{abstract}

Key words: basketball, dynamics, physical preparation, exercises

Introduction: Basketball is one of the popular team sports have received wide recognition both in the world and in Kazakhstan, which requires a manifestation of high physical, functional and emotional activity of the body of basketball player. For the game, characterized the work of variable intensity with a variety of cyclic motions walking, running, uniform and variable intensity. It is known that the basketball player in the game runs up to 4 miles, with performance of up to 150 accelerations and acyclic movements, stops, turns, jumps, for a total of up to 100 movements associated with fishing and shooting and dribbling, carried out in single combat with opponents that develop agility and coordination. All these features of the movements in basketball require a high anaerobic capacity in conditions of significant lack of oxygen and the development of a large bioenergy potential, as for the game, an athlete can lose up to 2-3 kilograms in weight.

Student age is the time when is most likely to implement sports performance in basketball [humenny V. S., 1; S. S. Yermakov, 2]. The highest results in student age are making basketball players, both in

\section{Kazakhstan and abroad.}

The problem of research is that the management of sports training in basketball requires the development of scientifically defensible indicators and the factors determining a potentially promising students - basketball players, able to acquire proficiency in a relatively limited period of a student's years of study at the University.

Modern basketball imposes extremely high requirements on the level of sporting achievements, requires continued improvement in the various forms of work with athletes at all stages of their athletic mastery [Egorychev A. O. et al. 3]. For achievement of high sports result, the underlying importance is the physical fitness level of the student, development of motor qualities (strength, speed, endurance, agility) and statistically significant combination of these qualities in accordance with the characteristics of the sport - basketball.. To solve this problem it is necessary to explore the factors that contribute to the achievement of high results in basketball, and in particular, to determine models for the respective 
stages of preparation and to evaluate the influence of levels of General and special physical fitness on the sport performance of students-basketball players.

It is known that exercise is more effective for the expansion of motor skills [Kaefer N.E., Solomeev S. A. Daurenbekov B.Z., 4] if they are used with regard to individual adaptation abilities of the organism, which determined the chosen direction of our research.

This article presents research and scientific study of the question of the influence of the training process subject to the control of the levels of physical training of student basketball teams in 2 mesocycles of speed-power orientation in the annual cycle of training.

The hypothesis of the study. It is expected that when included in the training process of students engaged in basketball, specific exercises speed-power orientation, can affect the development of physical qualities.

The purpose of the research is to investigate the dynamics of development of physical qualities in two mesocycles of the annual cycle to justify the rationality of a technique of development of speed-power qualities of students - basketball players

\section{Objectives of the study:}

1. Study of the dynamics of development, maximum speed, speed-strength and coordination qualities, a General and speed endurance basketball players are students in the annual macrocycle under the influence 2 mesocycles containing special exercises speed-strength character.

2. The rationale of rationality methods of development speed-power qualities of students who play basketball under the influence 2 mesocycles of special exercises speed-strength character.

Research methods. To solve the set goals and objectives, we have following methods were used: analysis of literature data, pedagogical observation, testing; method of mathematical statistics.

The results of the research. To implement the hypothesis and aims of the work in the experimental group investigated the effects of special complexes of exercises of speed and power focus on the development of physical qualities of students in the training process in basketball. For the control group training was carried out according to conventional standard program.
In the experimental group, in the first mesocycle, applied a complex of exercises directed on development of certain motor skills lagging muscle groups, and mastering the technique of special strength exercises with squats, jumps with a lightweight neck, work with dumbbells and weights. This complex means of speed-strength training was directed at the formation through the effect on the body exercises a mixed bio-energy intensity, primarily on the speed of a holistic motor skills development and special jumping.

The second mesocycle included the complex effects of the physical culture concentrated on the development of "specialized" physical qualities with emphasis on power training, which was aimed at improving the efficiency of game actions performed in the conditions of active confrontation.

The study of dynamics of development of physical qualities was conducted on 32 basketball players of high qualification (16 in the control and 16 in experimental group). This article presents two series of studies for the experimental group on the impact of the two mesocycles with a basic set of exercises with emphasis on developing speed and power qualities, lactate-anaerobic focus.

Pilot test was carried out every 2 months, after completing the training period a series of exercises of speed-power orientation, which was varied, based on the body's ability to adapt. The original data in the control and the experimental group was received in September 2016.

Subsequent tests were conducted after use of the recommended methodology for the development and improvement of power-speed players in the first microcycle, in November, and the second, in the month of January. The results of the test have been mathematics - statistical processing of finding the average value, percentage indicators of reliability of differences. Comparative analysis of statistical data was conducted between the experimental and control groups.

A study of baseline data on physical preparation at the beginning of the experiment in September 2016 showed that all the parameters of the control and experimental groups had average values of statistically significant differences.

Control of physical fitness in the experimental group of basketball players (table 1, figure 1) after 
1 mesocycle 2 months after the start of the experiment (November 2016) showed that players developed complexes of exercises of speed-power orien- tation was accompanied by a statistically significant increase of all studied traits.

Table 1 - Dynamics of indicators of physical readiness of the two mesocycles of speed-power orientation in the semiannual training cycle of students-basketball players of experimental group

\begin{tabular}{|c|c|c|c|c|c|}
\hline Tests Source & $\begin{array}{c}\text { indicators } \\
\text { (September) }\end{array}$ & $\begin{array}{c}\text { 1st mesocycle } \\
\text { (Nov) }\end{array}$ & $\begin{array}{c}\text { 2nd mesocycle } \\
\text { (January) }\end{array}$ & $\begin{array}{c}\text { 1st mesocy- } \\
\text { cle, } \%\end{array}$ & $\begin{array}{c}\text { 2nd mesocy- } \\
\text { cle, } \%\end{array}$ \\
\hline Running 20 meters, with & 3,58 & 3,52 & 3,43 & 1,78 & $4,19^{*}$ \\
\hline Running $60 \mathrm{~m}$, from & 8,19 & 8,12 & 8,06 & 0,85 & 1,56 \\
\hline $\begin{array}{l}\text { Running } 20 \mathrm{~m} \text { with dribbling, } \\
\text { with }\end{array}$ & 3,63 & 3,56 & 3,48 & 1,93 & $4,13^{\star}$ \\
\hline Move in defensive positions, with & 21,57 & 19,81 & 19,83 & $8,16^{*}$ & $8,07^{\star}$ \\
\hline Long jump, $\mathrm{cm}$ & 233 & 239 & 250 & 2,56 & $9,30^{*}$ \\
\hline Jump height, $\mathrm{cm}$ & 50 & 54 & 53 & $8,0^{*}$ & $6,0^{*}$ \\
\hline Shuttle running over $40 \mathrm{~m}$ & 252 & 259 & 267 & $2,78^{\star}$ & $5,95^{\star}$ \\
\hline The Cooper test, $\mathrm{m}$ & 2210 & 2220 & 2225 & 0,45 & 0,67 \\
\hline Serial jumping, & 13,05 & 12,00 & 11,20 & $8,05^{*}$ & $14,18^{*}$ \\
\hline Throwing the medicine ball, $\mathrm{m}$ & 8,76 & 9,06 & 9,34 & $5,42^{\star}$ & $6,62^{*}$ \\
\hline
\end{tabular}

A significant increase of the average data in the test, moving into a protective stance, reflecting the special physical speed and coordination ability, was $j$ 1.76 seconds or 8 , and $16 \%(\mathrm{p}<0.05)$; in the jump length of $6 \mathrm{~cm}$ or $2.57 \%(\mathrm{p}<0.05)$; in throwing the medicine ball 0.3 meters or $3.42 \%(\mathrm{p}<0.05)$; Shut- tle run for 40 seconds 7 meters, or $6.7 \%(\mathrm{p}<0.05)$; in the high jump of $4 \mathrm{~cm}$ or $8.0 \%(\mathrm{p}<0.05)$; serial jumping the 1.05 or $8,05 \%(\mathrm{p}<0.05)$ and false differences test Cooper $-10 \mathrm{~m}$ or $0.45 \%$; in run on 60 $\mathrm{m}$ with $-\mathrm{s}$ or 0.07 to $0.85 \%$; in run on $20 \mathrm{~m}$ with dribbling -0.07 with or $1.93 \%$.

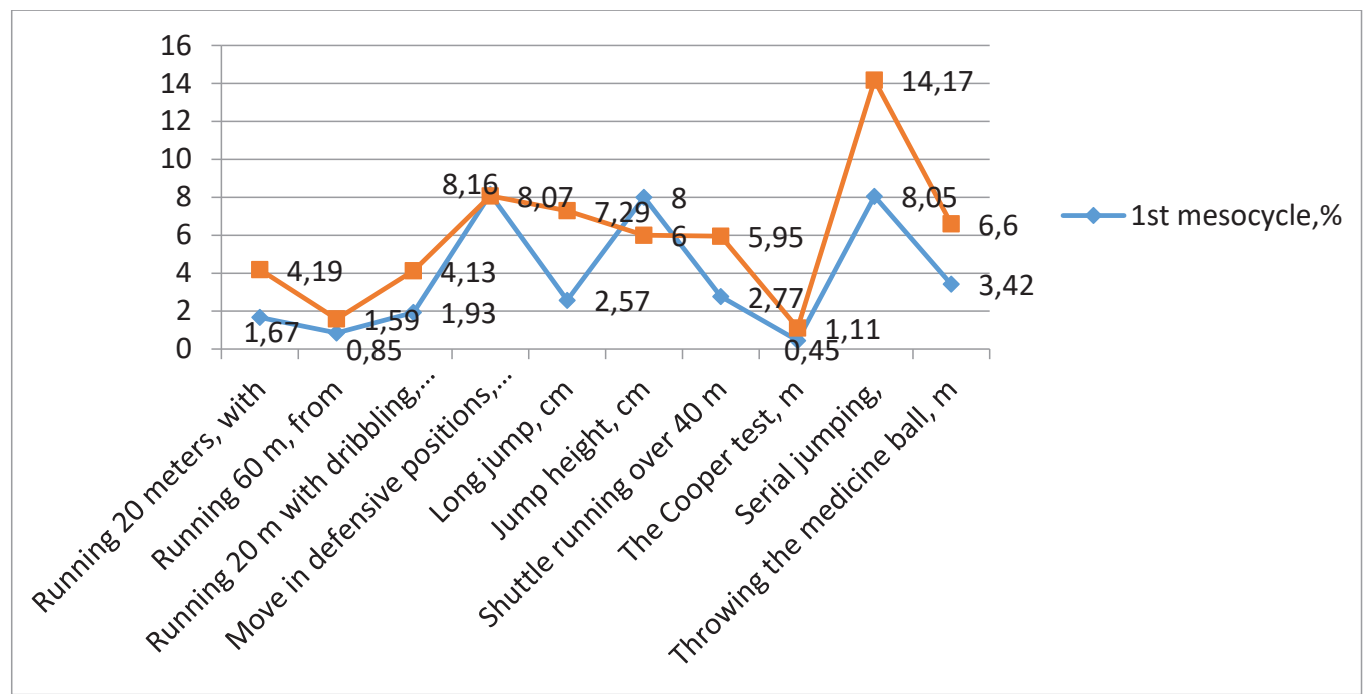

Figure 1 - Percent differences in levels of physical fitness of the students' basketball players of experimental group after 2 mesocycles of training of speed-power orientation

Thus, after mesocycle 1 , in the experimental group, using a combination of technical - tactical exercises with speed and strength training led to more significant changes in $50 \%$ of studied parameters particle velocity strength training. 
After the 2nd mesocycle in the experimental group had improved results in $80 \%$ of tests, such as: running on $20 \mathrm{~m}-0.09$ (4.19 percent); run for $20 \mathrm{~m}$ with dribbling - $0.08 \mathrm{~s}(4,13 \%)$; move into protective stand - by $0.98 \mathrm{C}(8,07 \%)$; long jump - $9 \mathrm{~cm}$ In the control group (table 2, figure 2), after 1 mes-

ocycle during the same periods as that for the experimental group, there was an increase, $\backslash$ indicators of physical fitness level of speed-power orientation only $50 \%$ tests performance: travel time in defensive 0.68 with an or of $3.52 \%(\mathrm{p}<0.05)$;

Table 2 - Dynamics of the control group average values of physical fitness in the semi-annual macrocycle preparation of students - basketball players

\begin{tabular}{|l|c|c|c|}
\hline \multicolumn{1}{|c|}{ Tests Sep, source } & $\begin{array}{c}\text { indicators (Septemt } \\
\text { ber) }\end{array}$ & $\begin{array}{c}\text { 1st mesocycle } \\
\text { (November) }\end{array}$ & $\begin{array}{c}\text { the 2nd mesocycle } \\
\text { (January) }\end{array}$ \\
\hline Running 20 meters, with & 3,66 & 3,55 & 3,54 \\
\hline Running 60 m, from & 8,17 & 8,16 & 3,17 \\
\hline $\begin{array}{l}\text { Running 20 m with dribbling, } \\
\text { with }\end{array}$ & 3,75 & 3,69 & 20,50 \\
\hline $\begin{array}{l}\text { Move in defensive positions, } \\
\text { with }\end{array}$ & 21,50 & 20,82 & 240 \\
\hline Long jump, cm & 230 & 236 & 52 \\
\hline Jump height, cm & 50 & 52 & 263 \\
\hline Shuttle running over 40 m & 250 & 255 & 2220 \\
\hline The Cooper test, $\mathrm{m}$ & 2201 & 12,90 & 9,26 \\
\hline Serial jumping ability, with, & 13,00 & 9,00 & \\
\hline Throwing the medicine ball, $\mathrm{m}$ & 8,71 & & \\
\hline
\end{tabular}

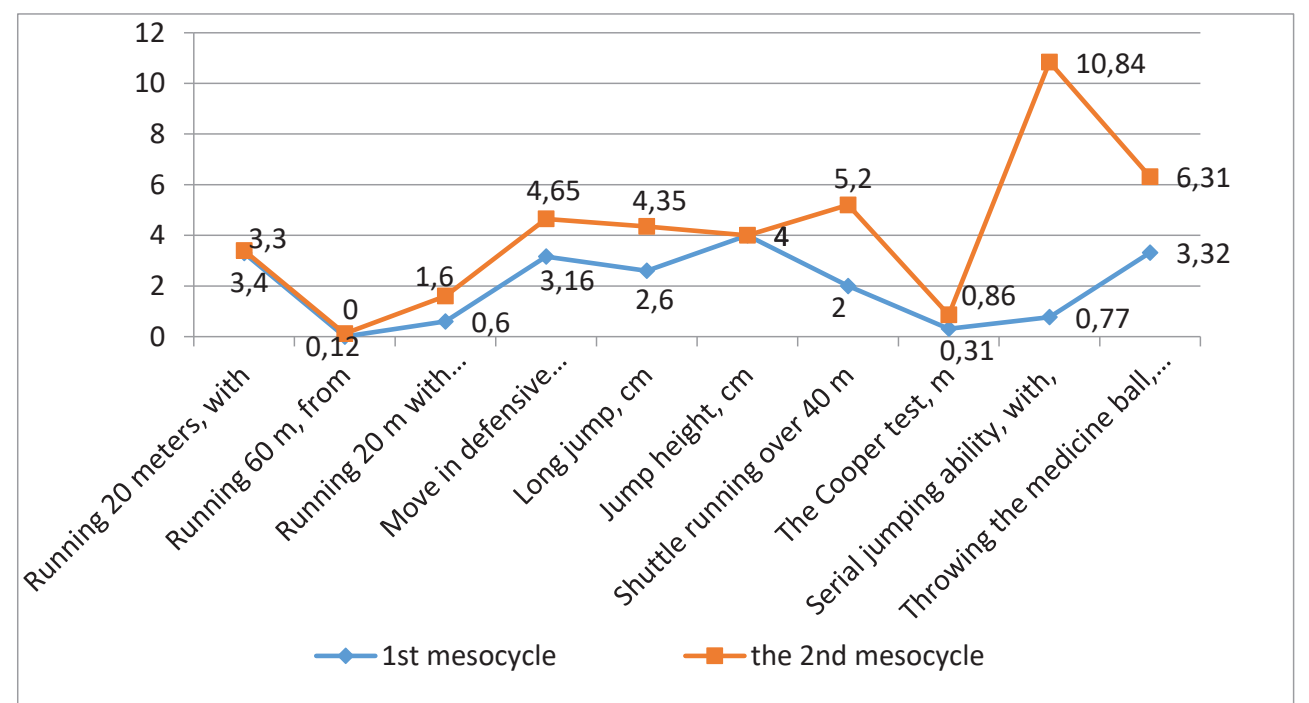

Figure 2 - Percentage differences of the levels of physical fitness of the students' basketball players of the control group

after 2 mesocycles of training of speed-power orientation throwing the medicine ball to $0.29 \mathrm{~m}$, which amounts to $3,31 \%$ ( $p<0.05)$; in the long jump by $6 \mathrm{~cm}$ or $2.57 \%$ $(p<0.05)$ and a height of 8.0 percent; Shuttle run $-5 \mathrm{~m}$ (6.7 percent).

At the end of mesocycle 2 a significant increase was observed in the indicators of serial jumping to $10.15 \%$ and Shuttle running over $403.14 \%$ Other indicators were lower in $70 \%$ of controlled tests.

Table 3 presents the percentage changes in the dynamics of the average indices of physical fitness of control and experimental groups in the semi-annual macrocycle preparation of students - basketball players. 
Table 3 - Changes in percent of the average indices of physical fitness of control and experimental groups in the semi-annual macrocycle preparation of students - basketball players

\begin{tabular}{|l|c|c|c|c|}
\hline \multicolumn{1}{|c|}{ Tests } & 1st meso-cycle\% & 2nd mesocycle,\% & 1st mesocycle,\% & 2nd mesocycle,\% \\
\hline & \multicolumn{2}{|c|}{ control group } & \multicolumn{2}{c|}{ experimental group } \\
\hline Running 20 meters, with & 3,00 & 3,00 & 1,67 & $4,19^{*}$ \\
\hline Running 60 m, from & 0,31 & 0,12 & 0,85 & 1,59 \\
\hline Running 20 m with dribbling, with & 1,6 & $-1,08$ & 1,93 & $4,13^{*}$ \\
\hline Move in defensive positions, with & 3,16 & 1,53 & 8,16 & $8,07^{*}$ \\
\hline Long jump, cm & 2,6 & 1,69 & 2,57 & $7,29^{*}$ \\
\hline High jump, cm & 4,0 & 0 & 8,0 & 6,0 \\
\hline Shuttle running over 40 m & 2,0 & $3,14^{*}$ & 2,77 & $5,95^{*}$ \\
\hline The Cooper test, m & 0,31 & 0,54 & 0,45 & 1,11 \\
\hline Serial jumping ability, with, & 0,77 & $10,15^{\star}$ & 8,05 & $14,17^{\star}$ \\
\hline Throwing the medicine ball, m & 3,33 & 2,69 & 3,42 & $6,6^{*}$ \\
\hline Note ${ }^{*}$ increased & \multicolumn{4}{|l}{} \\
\hline
\end{tabular}

The analysis of the presented data showed that in the control group positive dynamics of growth of indicators identified in $20 \%$ of tests and in the experimental group $80 \%$ of the tests, which confirms the greater efficiency of the applied exercises of speed and power orientation in comparison with a control series of studies.

Thus, the results of studies of the effectiveness of an improved method revealed a positive effect of experimental training on many of the indicators of physical development of students involved in basketball.

\section{Insights}

1. The use of special complexes of exercises of speed-power orientation with alternating volume and intensity in accordance with the specific planning of weekly training cycles, with three sessions per week has led to positive results.

Research conducted after the pilot trainings in 2 training mesocycles showed the greatest increase of speed strength training in the experimental group. Significant increase in speed-strength, coordination scores ranged from 4,13 to $14.17 \%$ ( $\mathrm{p}<0.05$ ), $80 \%$ of the tests: running at 20 meters, 60 meters and 20 meters with dribbling the ball, when moving into a protective stance, long jump, high jump, Shuttle run $2 \times 40$, except for indicators running on Cooper, reflecting the overall speed endurance, and in running on $60 \mathrm{~m}$, which was not observed statistically significant changes.

2. The results of the pedagogical experiment proved the effectiveness of used in the experiment methods of speed-strength training of students engaged in basketball, the mid-year macrocycle.

\section{References:}

Egorychev A. O., Pencic B.N., Bondarenko K. A., Smirnova, Y. A. Health students from a position of professionalism / // Theory and practice NAT. culture.2003.- N2.- P. 53-56.

Humenny V.S. To the problem of optimization of physical education of students of Polytechnic universities / V. S. humenny // Physical education of students of creative specialties / KSADA (hhpi).- Kharkov, 2002.- N7.- Pp. 63-72.

Iermakov S.S. Substantiation of pedagogical training shock movements in sports / S. S. Ermakov // Physical education of students of creative specialties / hhpi.Kharkov, 2001.- N3.- P. 24-29.

Kaefer N.E., Solomeev S. A. Daurenbekov B.Z. Special training of young basketball players $14-15$ years. // Theory and methods of physical culture.- 2015.- No. 2.- S. 91-102. 